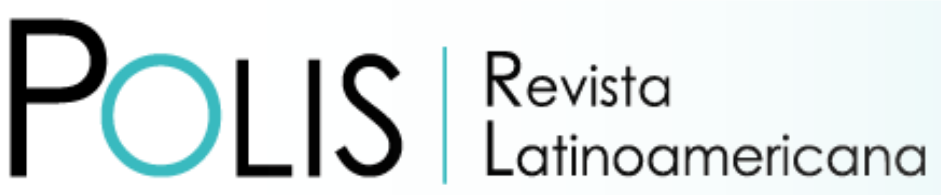

V20 | N58 | 2021

DOI: $10.32735 /$ S0718-6568/2021-N58-1576

\title{
Inclusión de derechos indígenas en Chile: Arquetipo constituyente desde América Latina
}

\author{
Carolina Acevedo De La Harpe \\ Universidad Católica de Temuco. Temuco, Chile \\ Email: cacevedo@uct.cl
}

Recibido: 31.08 .2020 | Aceptado: 02.12.2020

\begin{abstract}
Resumen: La experiencia Latinoamericana en el reconocimiento constitucional sobre derechos indígenas es fundamental para el proceso constituyente que se vivirá en Chile. Sobre la base de un análisis cualitativo comparativo (QCA) de 15 países se busca entender cómo los factores institucionales, políticos y sociales han sido determinantes para explicar un arquetipo de reconocimiento de derechos exitoso para los pueblos indígenas en América Latina.

Los resultados del análisis muestran que el tipo de mecanismo institucional para el cambio de la constitución, en particular la asamblea/convención constituyente y la participación de los movimientos indígenas son necesarios para explicar la obtención de mayores derechos indígenas en las constituciones. Este estudio busca aportar en la comprensión de los resultados de los procesos constituyentes a nivel comparado, así como brindar un diálogo académico propicio para el proceso constituyente en materia indígena que vivirá próximamente Chile.
\end{abstract}

Palabras Claves: Cambio Constitucional; Derechos Indígenas; América Latina; Proceso Constituyente; Asamblea Constituyente.

\section{Inclusion of Indigenous Rights in Chile: A Constituent Archetype from Latin America}

\begin{abstract}
The Latin American experience in the constitutional recognition of indigenous rights is fundamental for the constitutional process that will occur in Chile. Based on a comparative qualitative analysis (QCA) of 15 countries, we seek to understand how institutional, political and social factors have been determining factors to explain a successful archetype of recognition of rights for indigenous peoples in Latin America.

The results of the analysis show that the type of institutional mechanism for changing the constitution, in particular the constituent assembly / constitutional convention and the participation of indigenous movements are necessary to explain the obtaining of greater indigenous rights in the constitutions. This study seeks to contribute to the understanding of the results of the constituent processes at a comparative level, as well as to provide an academic dialogue conducive to the constituent process in indigenous matters that Chile will experience soon.
\end{abstract}


Keywords: Constitutional Change; Indigenous Rights; Latin America; Constituent Process; Constituent Assembly.

\section{Inclusão dos direitos indígenas no Chile: arquétipo constituinte desde a América Latina}

Resumo: A experiência latino-americana no reconhecimento constitucional dos direitos indígenas é fundamental para o processo constitucional que se viverá no Chile. A partir de uma análise qualitativa comparativa (QCA) de 15 países, buscamos compreender como fatores institucionais, políticos e sociais têm sido determinantes para explicar um arquétipo bem-sucedido de reconhecimento de direitos para povos indígenas na América Latina.

Os resultados da análise mostram que o tipo de mecanismo institucional de mudança da constituição, em particular a assembleia/convenção constituinte e a participação dos movimentos indígenas são necessários para explicar a obtenção de maiores direitos indígenas nas constituições. Este estudo busca contribuir para a compreensão dos resultados dos processos constituintes em nível comparativo, bem como propiciar um diálogo acadêmico conducente ao processo constituinte em matéria indígena que o Chile vivenciará em breve.

Palavras-chave: Mudança Constitucional; Direitos Indígenas; América Latina; Processo constituinte; Assembleia Constituinte.

\section{Como citar este artículo:}

Acevedo de la Harpe, C. (2021). Inclusión de derechos indígenas en Chile: Arquetipo constituyente desde América Latina. Polis Revista Latinoamericana, 20 (58), 22-42. doi: http:// dx.doi.org/10.32735/S0718-6568/2021-N58-1576

\section{Introducción}

El "estallido social" que se vivió en Chile durante octubre del 2019 activó a la ciudadanía en las calles. Más de 8 semanas de intensas y masivas' marchas, cacerolazos y diferentes expresiones que no dejaron indiferente a la comunidad internacional y por supuesto, a la clase política.

La respuesta institucional a la crisis social, política y económica que se instauró en la arena política, y que está marcando la agenda actual2, fue el acuerdo alcanzado por los partidos políticos para iniciar un proceso constituyente que permitirá redactar una nueva Constitución para Chile ${ }^{3}$. Esto, en el marco de una de las demandas que se ha levantado

\footnotetext{
1 Para un análisis profundo sobre el proceso de movilización ver Somma, Bargsterd, Disi y Medel (2020) "No water in the oasis: the Chilean Spring of 2019-2020". Social Movement Studies Journal.

2 Dos son los últimos hitos relacionados con el proceso constituyente. Primero, la aprobación de paridad de género en el órgano constituyente. Con esta aprobación, Chile sería el único país en el mundo en redactar una constitución con un antecedente de paridad determinado por ley. En segundo lugar, el día 20 de octubre con un $78 \%$ de aprobación la ciudadanía decide tras un plebiscito nacional comenzar el proceso constituyente, a través de una convención constituyentes.

3 Para detalles revisar: https://www.camara.cl/prensa/noticias_detalle.aspx?prmid=138442
} 
con mayor fuerza, derribar la constitución de 1980, heredada de la dictadura militar y que hoy hace eco como una de las principales razones de injusticia y desigualdad que se viven en Chile.

Diversos autores señalan que las expresiones de ilegitimidad y vulnerabilidad del sistema político incentivan a los ciudadanos a exigir y movilizarse en torno al aumento de derechos ciudadanos (véase, McAdam, 1996; Tarrow, 1998, entre otros). Dado este escenario, los sistemas políticos le asignan un valor especial a la constitución ya que sería el instrumento jurídico y político que se posiciona en la cima de toda regulación, donde se establecen aspectos estructurales como el sistema de gobierno, el poder, las funciones y limitaciones de las instituciones. Pero también, porque significa un nuevo contrato entre el Estado y la sociedad donde se involucran dimensiones simbólicas, de identidad e ideológicas (véase, Kelsen, 1945; Foweraker, 1995; Yrigoyen, 2000; Nolte y Schilling-Vacaflor, 2012; Galligan y Versteeg, 2013; Millaleo, 2019, entre otros).

En un Chile conflictuado históricamente con los pueblos indígenas y su nulo reconocimiento constitucional (véase, Bengoa, 1999; Salinas, 2005; Mella, 2007; Pairicán, 2013, Pinto, 2016; Figueroa, 2016, entre otros) surge la disyuntiva de cómo la demanda social y política podría reflejar en la convención constituyente la diversidad social/cultural del territorio. Sobre todo, si se considera que la nueva constitución guiará la agenda prioritaria de los próximos gobiernos y la forma de alcanzar esas metas, por ejemplo, la construcción de un Estado plurinacional, protección de la propiedad privada vs. derechos colectivos, entre otros ${ }^{4}$.

Este artículo busca aportar en el debate constitucional actual, comparando las experiencias de países de América Latina que han reconocido derechos indígenas en sus constituciones. Específicamente cómo y qué factores fueron claves en el alto reconocimiento de derechos indígenas. El argumento central sostiene que la asamblea/convención constituyente junto a una participación del movimiento indígena (mediante escaños reservados y participación de los movimientos sociales) dentro del proceso constituyente son claves para explicar un alto reconocimiento de derechos indígenas.

Se busca contribuir a una comprensión matizada de los resultados del reconocimiento constitucional indígena. Utilizando el método de análisis comparativo cualitativo (QCA) se comparan 15 países de América Latina con efectivo reconocimiento de derechos indígenas en las constituciones. Para responder la interrogante central se consideraron factores institucionales y sociales. el origen institucional o crisis del cambio constitucional; la inminente participación de movimientos indígenas en el levantamiento de la demanda, así como la asignación de escaños reservados para su participación; el mecanismo por el cual se lleva a cabo el cambio constitucional (asamblea/convención constituyente, legislación constituyente o reformas constitucionales).

4 Para mayor profundidad sobre los distintos derechos reconocidos en materia indígena en las constituciones véase Fuentes y Fernández (2020) 
Finalmente, los resultados y recomendaciones para Chile de cara a este momento político y social constituyente se enmarcan en los esfuerzos sociales e institucionales de los países Latinoamericanos que han alcanzado un alto grado de reconocimiento en materia indígena en sus constituciones. El arquetipo instaurado desde estas experiencias se basa en la promoción de una asamblea/convención constitucional plural, representativa y democrática incluyendo a los pueblos indígenas, a partir de diferentes formas de participación.

\section{América Latina: Arquetipo del reconocimiento constitucional indígena}

Desde 1990 aproximadamente 5 en América Latina comienzan a surgir movimientos indígenas que demandan reconocimiento y participación al Estado. La emergencia indígena acompañó los procesos de transformación del Estado en los distintos países, enfocándose en los cambios y reformas sobre las constituciones como eje central de la reingeniería institucional. Esta estrategia sobrevenida por una ventana de oportunidades ofrecida por las crisis de legitimidad o los cambios de régimen otorgó a las organizaciones y movimientos indígenas la posibilidad de expresar sus malestares y conseguir derechos constitucionales que los protegieran (véase, Van Cott, 2002, 2005; Bengoa, 2003; Martí i Puig, y Villalba 2012 , entre otros).

Estas nuevas constituciones o bien sus reformas incluyeron en muchos casos el reconocimiento de un conjunto de derechos sociales, culturales y ambientales, que fortalecieron los compromisos sociales entre el Estado y sus pueblos (Gargarella, 2013). Establecer un diálogo sobre el derecho consuetudinario proporcionó a los movimientos indígenas oportunidades políticas para el reconocimiento constitucional, aplicabilidad y protección más allá de los tratados internacionales. Como señalan distintos trabajos, las constituciones permitieron enfrentar un desajuste social en torno a un Estado unitario que no proyectaba un lugar para los pueblos indígenas (Yrigoyen, 2000; Scheppele, 2013; Gargarella, 2013, entre otros). No solo era una posibilidad de garantizar derechos y deberes entre sujetos olvidados o sub-incluidos (Millaleo, 2019), sino que también un espacio común hacia donde se debía avanzar como sociedad. Este marco de negociaciones interinstitucionales y de movilizaciones permitió pensar en una forma distinta de convivencia para los Estados y sus pueblos indígenas, una donde la orientación política del Estado reconociese mediante norma y política pública la diversidad cultural redefiniendo lo que se entendía por comunidad política, Estado-nación y convivencia social (véase, Moncusí ferré, 2004; Figueroa, 2006, Uprimny, 2014; Figueroa, 2019; Fuentes y Fernández, 2020, entre otros).

Los resultados variaron ampliamente en América Latina, en muchos países se puede hablar de constituciones plurinacionales (por ejemplo, Bolivia y Ecuador) debido a su amplio y explícito reconocimiento en derechos para los pueblos indígenas (véase, Bengoa, 2003; Martí i Puig y Villalba, 2012, entre otros) pero en otros casos se lograron escasos avances,

5 Bengoa, J. (2007), en su libro "La emergencia indígena en América Latina" presenta argumentos necesarios para poder entablar un contexto temporal del surgimiento de la temática. 
incluso aún existen países (como Chile o Guayana) donde la constitución no trata aspectos relativos a los pueblos indígenas. Si se toman los modelos de Van Cott (2000) y Aguilar, LaFosse, Rojas y Steward (2011) respecto de los derechos reconocidos en cada país, se puede encontrar desde derechos que hablan sobre la identidad, el territorio y la lengua, hasta constituciones que explicitan el carácter autónomo y social de los pueblos en miras a un Estado plurinacional ${ }^{6}$. En la Tabla 1 se ofrece un resumen de los países y de los distintos derechos según los modelos antes mencionados - 7 derechos Para Van Cott (2000) y 8 derechos para Aguilar, LaFosse, Rojas y Steward (2011). La diferencia entre ambos es que Van Cott (2000) en los derechos de propiedad colectiva incluye los derechos sobre los recursos naturales, aspecto que los segundos autores desagregan, por el contrario, estos últimos no consideran como derecho el reconocimiento del convenio 169, que es más bien un tratado internacional.

Tabla 1:

Países con reformas constitucionales y derechos indígenas reconocidos (1988-2011)

\begin{tabular}{|l|c|c|c|c|c|c|c|c|c|}
\hline & Púndigena & $\begin{array}{c}\text { Diversidad } \\
\text { Cultrual }\end{array}$ & $\begin{array}{c}\text { Tierra y } \\
\text { Territorio }\end{array}$ & $\begin{array}{c}\text { Recursos } \\
\text { Naturales }\end{array}$ & $\begin{array}{c}\text { Derecho de } \\
\text { libre } \\
\text { determinación }\end{array}$ & $\begin{array}{c}\text { Idiomas y } \\
\text { Lengua } \\
\text { Indigena }\end{array}$ & $\begin{array}{c}\text { Eduación } \\
\text { Bilingüe }\end{array}$ & $\begin{array}{c}\text { Participación } \\
\text { Política }\end{array}$ & $\begin{array}{c}\text { Derechos } \\
\text { Consuetudinarios }\end{array}$ \\
\hline Argentina (1994) & $\mathrm{Si}$ & $\mathrm{Si}$ & $\mathrm{Si}$ & $\mathrm{Si}$ & $\mathrm{No}$ & $\mathrm{No}$ & $\mathrm{Si}$ & $\mathrm{No}$ & $\mathrm{No}$ \\
\hline Bolivia (2009) & $\mathrm{Si}$ & $\mathrm{Si}$ & $\mathrm{Si}$ & $\mathrm{Si}$ & $\mathrm{Si}$ & $\mathrm{Si}$ & $\mathrm{Si}$ & $\mathrm{Si}$ & $\mathrm{Si}$ \\
\hline Brasil (1968) & $\mathrm{No}$ & $\mathrm{Si}$ & $\mathrm{Si}$ & $\mathrm{Si}$ & $\mathrm{No}$ & $\mathrm{Si}$ & $\mathrm{No}$ & $\mathrm{No}$ & $\mathrm{No}$ \\
\hline Chile (1980) & $\mathrm{No}$ & $\mathrm{No}$ & $\mathrm{No}$ & $\mathrm{No}$ & $\mathrm{No}$ & $\mathrm{No}$ & $\mathrm{No}$ & $\mathrm{No}$ & $\mathrm{No}$ \\
\hline Colombia (1991) & $\mathrm{Si}$ & $\mathrm{Si}$ & $\mathrm{Si}$ & $\mathrm{Si}$ & $\mathrm{Si}$ & $\mathrm{Si}$ & $\mathrm{Si}$ & $\mathrm{Si}$ & $\mathrm{Si}$ \\
\hline Costa Rica (2014) & $\mathrm{No}$ & $\mathrm{Si}$ & $\mathrm{No}$ & $\mathrm{No}$ & $\mathrm{No}$ & $\mathrm{Si}$ & $\mathrm{No}$ & $\mathrm{No}$ & $\mathrm{No}$ \\
\hline Ecuador (2008) & $\mathrm{Si}$ & $\mathrm{Si}$ & $\mathrm{Si}$ & $\mathrm{Si}$ & $\mathrm{Si}$ & $\mathrm{Si}$ & $\mathrm{Si}$ & $\mathrm{Si}$ & $\mathrm{Si}$ \\
\hline El Salvador (1992) & $\mathrm{No}$ & $\mathrm{Si}$ & $\mathrm{No}$ & $\mathrm{No}$ & $\mathrm{No}$ & $\mathrm{Si}$ & $\mathrm{No}$ & $\mathrm{No}$ & $\mathrm{No}$ \\
\hline Guatemala (1994) & $\mathrm{No}$ & $\mathrm{Si}$ & $\mathrm{Si}$ & $\mathrm{No}$ & $\mathrm{No}$ & $\mathrm{Si}$ & $\mathrm{Si}$ & $\mathrm{No}$ & $\mathrm{No}$ \\
\hline Honduras (1982) & $\mathrm{No}$ & $\mathrm{Si}$ & $\mathrm{No}$ & $\mathrm{No}$ & $\mathrm{No}$ & $\mathrm{No}$ & $\mathrm{No}$ & $\mathrm{No}$ & $\mathrm{No}$ \\
\hline México (1992) & $\mathrm{Si}$ & $\mathrm{Si}$ & $\mathrm{Si}$ & $\mathrm{Si}$ & $\mathrm{Si}$ & $\mathrm{Si}$ & $\mathrm{Si}$ & $\mathrm{Si}$ & $\mathrm{Si}$ \\
\hline Nicaragua (2008) & $\mathrm{Si}$ & $\mathrm{Si}$ & $\mathrm{Si}$ & $\mathrm{Si}$ & $\mathrm{Si}$ & $\mathrm{Si}$ & $\mathrm{Si}$ & $\mathrm{Si}$ & $\mathrm{Si}$ \\
\hline Panamá (2008) & $\mathrm{No}$ & $\mathrm{Si}$ & $\mathrm{Si}$ & $\mathrm{No}$ & $\mathrm{No}$ & $\mathrm{Si}$ & $\mathrm{Si}$ & $\mathrm{Si}$ & $\mathrm{No}$ \\
\hline Paraguay (1992) & $\mathrm{Si}$ & $\mathrm{Si}$ & $\mathrm{Si}$ & $\mathrm{No}$ & $\mathrm{Si}$ & $\mathrm{Si}$ & $\mathrm{Si}$ & $\mathrm{Si}$ & $\mathrm{Si}$ \\
\hline Perú (1993) & $\mathrm{No}$ & $\mathrm{Si}$ & $\mathrm{Si}$ & $\mathrm{No}$ & $\mathrm{Si}$ & $\mathrm{Si}$ & $\mathrm{Si}$ & $\mathrm{No}$ & $\mathrm{Si}$ \\
\hline Venezuela (1999) & $\mathrm{Si}$ & $\mathrm{Si}$ & $\mathrm{Si}$ & $\mathrm{Si}$ & $\mathrm{No}$ & $\mathrm{Si}$ & $\mathrm{Si}$ & $\mathrm{Si}$ & $\mathrm{Si}$ \\
\hline
\end{tabular}

Fuente: Elaboración propia a partir de Van Cott (2000) y Aguilar, LaFosse, Rojas y Steward (2011)

Sin duda, los cambios o reformas constitucionales han formado un marco idóneo para reconocer y profundizar los procesos de autonomía, territorio y cultura indígena. Tal como se señaló al comienzo, existen países que han avanzado mucho en esta materia - México (1992), Nicaragua (2008), Bolivia (2009) o Ecuador (2008)- pero existen otros que no han podido dar grandes pasos -Honduras (1982), Costa Rica (2014) o Brasil (1988)-. Tal como explica Bengoa (2003), en muchos de los casos de éxito las constituciones se han visto obligadas a cambiar su estricta visión unitaria del Estado, esto producto de la entrada en el juego

6 Si bien no es menester de esta investigación evaluar los alcances de estos reconocimientos, es efectivamente interesante observar cómo los distintos procesos sociales, institucionales y políticos abordados en cada país derivaron en distintos niveles de reconocimiento de derechos indígenas. 
político del actor indígena, pero en otros casos los procesos han sido más laxos y han generado menos reconocimientos, lo que involucra procesos de negociación más complejos que son interesantes de observar.

Frente a este proceso han surgido diversos estudios que posicionan la temática, por ejemplo, el estudio de Martí i Puig y Villalba (2012) elabora un marco evaluativo respecto de los países de América Latina con menor población indígena y cómo su estructura de oportunidades les ha brindado apoyo para obtener mayores reconocimientos. Concluyendo que la constitución abarcó mayores aspectos culturales en los países donde el origen del cambio fue producto de una crisis de legitimidad del sistema y/o donde los movimientos indígenas adquirieron un carácter de actor relevante en el debate político. Lo interesante de su planteamiento para esta investigación es que se justifica la variabilidad en el reconocimiento, a través de los países y atribuye al sistema político y las estrategias de los actores estas diferencias. Van Cott (2000) por su parte, estudia las constituciones de 4 países del sector andino, concluyendo dos asuntos importantes para este estudio, por un lado, que el éxito en Colombia (1991) es fruto del movimiento indígena, el cual tuvo miembros "claves" en el proceso constituyente. Por otro lado, el éxito en Venezuela (1999) fue producto de la alianza con el presidente Hugo Chávez. Por tanto, lo que acá se busca es tomar la historia como argumento revelador de la dinámica de la política interna de cada país y observar qué factores son los relevantes para los derechos de los pueblos indígenas.

La temporalidad también es otro factor clave que no ha logrado predecir resultados respecto del alcance de derechos en las constituciones. La literatura habla de diferentes olas de reconocimiento: las primeras reformas (1988-1994) consideran a Brasil (1988), Colombia (1991), Paraguay (1992), México (1992), Guatemala (1994), Perú (1993) y Argentina (1994). La segunda ola reformista (1999-2004) fue en Venezuela (1999) y Panamá (2004). Por último, la tercera ola y la que quizás más renombre ha tenido por el nivel de reconocimiento, es la que surge desde el 2005 a la fecha en Bolivia (2009), Ecuador (2008), Nicaragua (2008), Costa Rica $(2014)^{7}$.

\section{Revisión de la literatura: Origen del cambio, mecanismos institucionales y movimientos indígenas}

La importancia que tienen las constituciones para la política institucional en América Latina se relaciona con los constantes procesos de cambios y las especificaciones en torno a marcos regulatorios y de acción jurídica, que modelan deberes y derechos, así como procesos de inclusión y coordinación de la sociedad (véase, North y Weingast, 1989; Weingast, 2005; Negretto, 2008, 2012; Gargarella, 2012, Millaleo 2019, entre otros). Específicamente, en América Latina estos procesos de coordinación, estabilización y cambio responden a una lógica institucional particular del entramado político y social que viven los distintos países, así como

7 Elaboración propia a partir de la información obtenida en el Seminario Internacional- Ciudadanía Indígena: Retos y nuevos desafíos para la Institucionalidad Pública (2013) 
lógicas de cooperación e inclusión/exclusión. Negretto (2008) señaló que la estabilidad de las constituciones es un atributo de una constitución creada como un dispositivo de coordinación eficaz, por tanto, la resolución exitosa de los conflictos surge de entre los responsables de la constitución sobre disposiciones constitucionales alternativas. Un ejemplo claro, es la existencia de problemas sobre reconocimiento de diferentes culturas en un mismo país, la coordinación entre la élite política y los ciudadanos es clave, pero no sencillo de resolver. La falta de inclusión por medio de la ciudadanía y reconocimiento de derechos también tiene por consecuencia problemas de reconocimiento en la interacción cotidiana de los sujetos (Millaleo, 2019), aspecto que amenaza constantemente las instituciones producto de esta exclusión. Weingast (1997), ya señalaba que las sociedades con divisiones de carácter económico, étnico o religioso a menudo sufren de inestabilidad constitucional crónica.

En cuanto a los niveles de reconocimiento, este puede variar desde aspectos genéricos de la diversidad cultural, pasando por niveles de multiculturalismo hasta un reconocimiento más profundo de Estado plurinacional (Yrigoyen, 2011). Por este motivo, la coordinación y estabilidad no es fácil, sobre todo considerando que los aspectos simbólicos que permean a los movimientos indígenas son contrarios a la idea de Estado-nación homogéneo. Sin embargo, frente a estas problemáticas se han buscado soluciones que parecieran ser homogéneas -reconocimientos de derechos en las constituciones- pero que finalmente han terminado con resultados muy diferentes. ¿Qué explica entonces los diferentes niveles de reconocimiento de derechos indígenas dentro de las constituciones de América Latina? Esta investigación alude a distintos factores institucionales y sociales que, a la luz de los casos, se han presentado como variables relevantes para explicar un camino de alto reconocimiento en materia indígena.

\section{Origen del cambio/reforma constitucional ¿Voluntad institucional o Crisis social?}

La literatura ha discutido que el origen del cambio/reforma puede comenzar producto de una voluntad institucional, es decir, por moción del ejecutivo o parlamento, o bien producto de una crisis política, económica y/o social. Desde la vereda institucional se ha posicionado al ejecutivo y al legislativo - principalmente los partidos que han logrado acuerdos- como los artífices o motor del cambio/reforma a la constitución.

La literatura es ambigua sobre el efecto que esto acarrea en materia de derechos indígenas, sin embargo, si se analiza en detalle el efecto institucional, se puede desprender que el ejecutivo se mantendría más bien neutral frente a estos procesos (véase, Negretto, 2008; Lijphart, 1993; Weyland, 2006; Knight, 1992; Weingast, 2005; North y Weingast, 1989, entre otros). Incluso es posible asumir que el rol de los presidentes es contradictorio a una reforma o cambio constitucional, ya que tiende a dividir el poder entre los actores. O bien, tal como señalan Pérez-Liñán (2010) y Cheibub (2007) la coordinación entre ejecutivo y legislativo se vuelca difícil y poco probable para generar este tipo de reformas o cambios, producto de la exagerada posición de poder del ejecutivo. 
Referente al poder legislativo, la literatura ha girado más en torno a los partidos políticos que acceden a los escaños parlamentarios producto de que programáticamente han ofrecido cambios o reformas a la constitución (Negretto, 2013), lo que posteriormente se ha traducido en negociaciones institucionales. Pero también se ha discutido cómo ciertos sectores políticos se han posicionado para la aprobación de legislación y romper el denominado gridlock actuando como actor pivotal (Krehbiel, 1991).

Finalmente, otro de los orígenes han sido las crisis sociales, políticas o económicas. Los casos muestran cómo el poder legislativo y/o ejecutivo reaccionan posteriormente a la exigencia ciudadana tras intensas manifestaciones (Bolivia 2008) o acciones coordinadas (Colombia 1991). El estudio de Martí i Puig y Villalba (2012) es clave para comprender este argumento. Los autores elaboran un marco evaluativo respecto de los países de América Latina con menor población indígena y cómo su estructura de oportunidades les ha brindado apoyo para obtener mayores reconocimientos. Concluyendo que la constitución abarcaría mayores aspectos culturales en los países donde se hubiera producido un cambio constitucional a raíz de una crisis de legitimidad del sistema, entre otras variables.

\section{Rol de los movimientos indígenas en los procesos constituyentes}

Los procesos constituyentes han presentado un espacio ideal para la acción colectiva, en especial para los grupos marginados y débilmente institucionalizados como los pueblos indígenas (Van Cott, 2002). La performance se da gracias a los espacios internos y externos del órgano constituyente -particularmente la asamblea constituyente- donde es posible participar activamente y beneficiarse del proceso de transformación alcanzando la incorporación política de derechos (mayor o menor grado), así como una mayor movilización-coordinación de grupos previamente excluidos.

Si bien no existe un acuerdo en la literatura, es posible identificar en esta lógica dos formas de contribución al reconocimiento de derechos por parte de los movimientos indígenas. Primero, están los partidos indígenas posicionando la temática y cooperando en la negociación para el reconocimiento de derechos.

Pero también está la posibilidad de que el movimiento a través de las distintas instancias de participación y/o en movilizaciones sociales actúe posicionando la temática en la agenda de los constituyentes (Martí i Puig y Villalba, 2012). En efecto, los movimientos indígenas que a pesar de no estar formalmente constituidos como partido político han participado como constituyentes o como actores que levantan las demandas y apoyan el proceso en conjunto a los movimientos sociales en pro de alcanzar el reconocimiento (se excluye el caso de Bolivia, país que alcanzó un nivel de organización política previa que fomentó enormemente los resultados). 
Respecto de la participación como constituyentes, la experiencia comparada ha mostrado diferentes formas de acceder a al cargo de representante, en algunos casos se ha logrado vía escaños reservados para pueblos indígenas y en otros países a través de formas distritales de elección especialmente diseñadas para pueblos indígenas. Sobre los escaños reservados para los pueblos indígenas, se han desarrollado varios argumentos a favor de los mecanismos de representación diferenciados por grupos, esto con la finalidad de ayudar a los grupos históricamente marginados a expresarse y acceder a un grado de influencia política (Young, 1990; Kymlicka, 2009; Mansbridge, 1999; Bird 2014, entre otros). Por otro lado, también se han diseñado mecanismos institucionales que flexibilizan la entrada de partidos o actores indígenas a los procesos constitucionales, por ejemplo, diseñando distritos electorales con perspectiva étnica o levantando barreras de entrada al sistema electoral. Todo lo anterior con la finalidad de equilibrar y/o dar igualdad al proceso considerando las características diversas de la sociedad (Bird, 2014).

Se ha discutido también sobre el momento en el que se interviene dentro del proceso constitucional, ya sea antes y/o durante el proceso. En este sentido, Ghai (2006) señala que cuando hay aspectos religiosos o étnicos involucrados dentro de las divisiones que componen el proceso de reforma o cambio constitucional las etapas de movilización previas son fundamentales para llegar a acuerdos. En la misma línea Negretto (2008) señala que incluso cuando los partidos o movimientos sociales participan del proceso, logran generar cambios por su participación como actor pivotal en acuerdos previos básicos, asunto que no estaría determinado por características como el tamaño del partido.

Lo antes expuesto, reafirma el argumento de que los determinantes del avance en materia de reconocimiento de derechos de los pueblos indígenas parecen relacionarse con la existencia de movimientos indígenas que, en coyunturas claves, logran incorporar la temática en el centro del debate político-constitucional, así como participar de forma efectiva en el proceso. Tal como se señala el texto de FORD y PNUD (2013), esto sucedió en Colombia (1991), Bolivia (2008), Ecuador (2008) o Venezuela (1999).

\section{Tipos de mecanismos utilizados para cambiar o reformar la constitución}

A nivel internacional una gran cantidad de países ha cambiado o modificado sus constituciones. Tal como señalan Ginsburg, Melton y Elkins (2010) la esperanza de vida de una carta magna es de aproximadamente 19 años, por lo tanto, los cambios y modificaciones son una regla más que una excepción. En tal escenario, América Latina no está ajena a dicha realidad, distintos procesos de cambio y reforma se han vivido en los últimos 30 años.

Bajo este escenario los mecanismos institucionales para elaborar una nueva constitución o realizar reformas han girado en torno a la creación de órganos específicos con mandato de redactar un nuevo texto. Como han sido las asambleas o convenciones constituyentes, pero también se pueden encontrar casos donde el poder legislativo ha adquirido dicha 
tarea. De los 15 países analizados cuatro han generado reconocimiento de derechos indígenas mediante reformas a la constitución vigente - Argentina (1994), Costa Rica (2014), Guatemala (1994) y Panamá (2004)- los demás han generado procesos de cambios completo en la constitución. De aquellos que han cambiado completamente, 6 países han ocupado la asamblea/convención constituyente como órgano de redacción - Bolivia (2009), Colombia (1991), Ecuador (2008), Honduras (1982), México (1992) y Venezuela (1999) - y 5 casos han realizado el cambio mediante un congreso constituyente - Brasil (1988), El Salvador (1992), Nicaragua (2008), Paraguay (1992) y Perú (1993)-.

La variación en el mecanismo institucional empleado permite observar que no existe una única forma exitosa para obtener mayores derechos indígenas. A pesar de lo empírico y los distintos estudios que han posicionado a la asamblea/convención constituyente como la forma idónea para alcanzar mayor legitimidad democrática y promover un diseño impar$\mathrm{Cial}^{8}$. Es interesante manifestar que los procesos institucionales no tienen una regla exclusiva, y que tal como señala Negretto (2018) todo va a depender del contexto que se de en el proceso propio de la elaboración de la constitución.

Sobre las legislaturas constituyentes, se puede señalar que estas han funcionado como legislatura ordinaria conjuntamente reforman/cambian la constitución. Según lo señalado por Negretto (2018) y lo registrado por PNUD (2014), las legislaturas constituyentes serían el mecanismo más utilizado, el cual además puede variar en su formato (puede ser una sola parte de la legislatura la que participe del proceso o formarse comisiones especiales) y en la forma de ratificación (congreso pleno, vía referéndum ciudadano o ambos). Más allá de los aspectos formales, la diferencia sustancial es que las legislaturas constituyentes son cuerpos que se auto crean para realizar esta tarea, diferenciándose de procesos sociales y/o basados en el poder constituyente originario (Negretto, 2018).

Finalmente, en América Latina el reconocimiento constitucional vía reforma ha sido escaso, pero no nulo. Por tanto, no se puede asumir que el simple hecho de elegir uno u otro mecanismo determinaría un alto nivel de reconocimiento. Las diferencias que puedan generarse producto de la forma en que se genera el cambio/reforma utilizada en esta investigación, son tan relevantes como el rol del ejecutivo y de los movimientos sociales. Ya que no sólo son posibilidades que el país tiene para organizarse, sino que en muchos casos activan el poder constituyente emanado desde el pueblo.

\section{Diseño de Investigación}

Se estudiarán países de América Latina que han realizado algún grado de reconocimiento de derechos indígenas en sus constituciones desde 1988 hasta 2015 (fecha de las últimas

8 Negretto (2018) enfatiza la relación entre asamblea constituyente y mejores resultados para la democracia y pluralidad, apuntando a que las preferencias ciudadanía se verían mejor reflejadas, así como una distribución de poder más equilibrada 
modificaciones constitucionales en materia indígena). Producto del interés central de la investigación los países y años de cambio/reforma seleccionados son: Argentina (1994), Bolivia (2009), Brasil (1988), Colombia (1991), Costa Rica (2014), Ecuador (2008), El Salvador (1992), Guatemala (1994), Honduras (1982), México (1992), Nicaragua (2008), Panamá (2004), Paraguay (1992), Perú (1993) y Venezuela (1999).

Respecto de la metodología, se realizará un análisis comparativo cualitativo denominado fuzzy set (fs-QCA), ya que los casos poseen un control interno y la posibilidad de ser calibrados tal como lo recomienda Ragin (2008). Por lo tanto, la posibilidad de generar configuraciones explicativas que se requieren para dar cuenta de un resultado es posible acorde a los datos y casos. Este método en particular se utiliza en estudios orientados a analizar pocos casos ( $n=15$ países) pero con un análisis intensivo, es decir, se hace cargo de muchos aspectos de los casos de forma sistémica. Como señalan Epstein, Kenworthy y Ragin (2008) la teoría de conjunto permite un nivel de error y de una visión del mundo probabilístico, al igual que el análisis estadístico de casos grandes. Presentando la posibilidad de realizar una estrategia analítica que tiene fines no solamente descriptivos, sino también explicativos, lo cual sirve para poner a prueba las hipótesis teóricas planteadas. Por otro lado, en fs-QCA (la versión crisp-set no lo necesita) los conjuntos de datos y variables explicativas deben ser calibrados en función de los resultados del estudio (para detalles de los valores originales y calibrados ver Tabla 1).

Respecto de la operacionalización de las variables, es posible señalar lo siguiente. La variable dependiente: nivel de derechos indígenas reconocidos en las constituciones se construyó en base a los estudios realizados por Van Cott (2000) y Aguilar et al. (2011). La variable asumió el porcentaje de derechos reconocidos por cada país en base a un total de 9 derecho presentados en la Tabla 1. Para calibrar los resultados se realizó un índice simple que va de 0 a 100 dependiendo de la cantidad de derechos constitucionales reconocidos. Se realizó un corte aritmético en 0 (bajo)- 50 (medio) - 100 (alto) para poder calibrar la variable, así los países al ser solo casos positivos quedan en rangos de entre el $20 \%$ y el $100 \%$.

Para la variable origen del cambio/reforma de la constitución. Se consideró si el proceso de cambio/reforma se origina desde una negociación institucional (desde el ejecutivo o parlamento) o bien desde una crisis (social, política o económica). Los valores otorgados son ( 1 = institucional; $0=$ crisis).

La participación de los movimientos indígenas se desagrego en dos variables. Por una parte, se consideró el porcentaje de población indígena del país, donde se realizó un corte aritmético en 0 (bajo)- 50 (medio) -100 (alto) para poder calibrar la variable, así los países al ser solo casos positivos quedan en rangos entre $1 \%$ y $65 \%$. En segundo lugar, se creó un indicador donde se registran tres formas de participación del movimiento indígena asignando valores de 0 (bajo)- 50 (medio) -100 (alto). Para el valor 100, se consideró si el país contó con escaños reservados para pueblos indígenas o algún diseño electoral especial, además de pesquisar si el movimiento indígena participó como actor clave en las negociaciones 
y/o procesos participativos. Para el valor 50 , se consideraron los casos donde el movimiento indígena participó como actor clave en las negociaciones y/o procesos participativos, pero sin contar con un mecanismo institucional de participación (por ejemplo, escaños reservados) ${ }^{9}$. Finalmente existen países donde no se contó con ninguna de las dos formas de participación, los que fueron asignados con valor 0.

Por último, está la variable mecanismos institucionales para el cambio/reforma de la constitución. Acá se generó un índice donde se otorgó puntaje mayor a la asamblea constituyente, dada la hipótesis de la literatura, principalmente considerando lo señalado por Negretto (2018), luego al parlamento constituyente y finalmente a una reforma de la constitución. Calibrando en 0 (bajo)- 50 (medio) -100 (alto) respectivamente las distintas figuras institucionales para el cambio/reforma constitucional.

\section{Resultados Empíricos}

Siguiendo los pasos de fs/QCA recomendados por Schneider y Wagemann (2012) este artículo realiza una prueba de necesidad por separado antes del análisis de suficiencia. Primero examina si cada una de las cuatro variables puede considerarse como una condición necesaria para un alto reconocimiento de derechos indígenas. Tal como se puede observar en la Tabla 2 ninguna de las condiciones propuestas cumple con el criterio de alcanzar una consistencia superior a 0.90 (Schneider y Wagemann 2012, p. 27), por lo que ninguna de las variables por sí sola explicaría el fenómeno. Sin embargo, las variables que asumen mayor consistencia son: participación de movimientos indígenas y mecanismo institucional para el cambio/reforma de la constitución.

Tabla 2:

Análisis de condición necesaria para casos con alto reconocimiento de derechos indígenas

\begin{tabular}{|l|c|c|}
\hline Condición & Consistency & Coverage \\
\hline Porcentaje Población Indígena & 0.20 & 0.99 \\
\hline Origen del cambio/reforma (institucional o crisis) & 0.60 & 0.52 \\
\hline Participación movimientos indígenas & 0.70 & 0.89 \\
\hline Mecanismo institucional para el cambio/reforma constitucional & 0.68 & 0.82 \\
\hline
\end{tabular}

Fuente: Elaboración propia.

Elanálisis deminimizaciónbooleanaquesemuestraenlatabladelaverdad (vertabla3) entrega la relación entre las variables propuestas y los resultados empíricos. A priori se puede observar que existen dos configuraciones causales que conducen a un alto reconocimiento de

9 No existen casos contrarios, es decir, donde si existen mecanismos institucionales como los escaños reservados y no existe participación del movimiento indígena. 
derechos indígenas, con un puntaje de consistencia de 1.00 y 0.92 respectivamente. Esto sugiere que las cuatro variables propuestas logran diferenciar los casos según sus distintos niveles de reconocimiento de derechos, y además no existieron casos contradictorios.

Tabla 3:

Tabla de la verdad casos con alto reconocimiento de derechos indígenas

\begin{tabular}{|c|c|c|c|c|c|c|c|}
\hline \multicolumn{6}{|c|}{ Tabla de la Verdad } \\
\hline $\begin{array}{c}\text { Población } \\
\text { Indígena }\end{array}$ & $\begin{array}{c}\text { Mecanismo } \\
\text { Institucional }\end{array}$ & $\begin{array}{c}\text { Origen del } \\
\text { cambio/reforma }\end{array}$ & $\begin{array}{c}\text { Participación } \\
\text { del movimiento } \\
\text { Indígena }\end{array}$ & $\begin{array}{c}\text { Derechos } \\
\text { Reconocidos }\end{array}$ & $\begin{array}{c}\text { Número de } \\
\text { casos }\end{array}$ & Casos & Consistencia \\
\hline 0 & 1 & 0 & 1 & 1 & 2 & $\begin{array}{c}\text { colombia } \\
\text { Ecuador }\end{array}$ & 1 \\
\hline 1 & 1 & 0 & 1 & 1 & 1 & Bolivia & 1 \\
\hline 0 & 1 & 1 & 1 & 1 & 2 & Venezuela & 0.84 \\
\hline
\end{tabular}

Fuente: Elaboración propia.

La minimización booleana arrojó una solución compleja con dos configuraciones causales para un alto reconocimiento de derechos indígenas (ver Tabla 4) ${ }^{10}$. Es preciso recordar que alto reconocimiento es un rango de entre 6 a 9 tipos de derechos reconocidos. La primera configuración muestra los resultados para los casos de Bolivia (2008), Colombia (1991) y Ecuador (2008). Países donde el origen del proceso constituyente comienza por una crisis política/social, la cual es canalizada mediante una asamblea constituyente y donde la participación del movimiento indígena se vincula a una participación en los procesos antes y durante el cambio, así como la creación de escaños reservados para los pueblos indígenas y/o un diseño electoral que favoreció el ingreso de los partidos en la constituyente.

El segundo resultado arrojó una configuración causal para los casos de Ecuador (2008), Colombia (1991) y Venezuela (1999). La diferencia sustancial con la primera configuración es que se elimina el caso de Bolivia e ingresa el caso de Venezuela (1999) por dos variables. Primero, porque Venezuela cuenta con un bajo porcentaje de población indígena. En segundo lugar, porque el origen de su proceso constituyente se originó por negociaciones institucionales, principalmente desde el presidente Hugo Chávez. Sumado a lo anterior, es importante señalar que la comparación de las dos configuraciones muestra que el mecanismo institucional para el cambio/reforma de la constitución y la participación del movimiento indígena aparecen en ambos resultados, lo que es consistente con el resultado del análisis de necesidad de la tabla 2.

Estos hallazgos coinciden con las propuestas de Van Cott (2001), Martí i Puig y Villalba (2012) y Negretto (2018) sobre las cualidades importantes de la asamblea constituyente, así como el rol de la participación de los grupos indígenas en la obtención de mayores derechos

10 Es importante para interpretar los resultados de la tabla entender que las variables con el signo ( ) significan que el valor 0 asumen la condición para el resultado. El signo $(*)$ significa que es la conjunción de las variables la que permite entender el resultado. 
reconocidos en la constitución. En las siguientes subsecciones, se tomarán los casos de Colombia (1991) y Bolivia (2009) para narrar con mayor detalle las configuraciones causales. Estos casos fueron seleccionados por su diferencia esencial en el porcentaje de población indígena, variable que pudiese ser clave para la comparación con el proceso chileno.

Tabla 4:

Solución Compleja QCA para casos con alto reconocimiento de derechos indígenas en constituciones

\begin{tabular}{|c|c|c|c|}
\hline & Casos & $\begin{array}{c}\text { Raw } \\
\text { Coverage }\end{array}$ & Consistency \\
\hline $\begin{array}{c}\text { Mecanismo institucional * Origen del cambio/reforma * } \\
\text { Participación movimiento indígena }\end{array}$ & $\begin{array}{c}\text { Bolivia, } \\
\text { Colombia, } \\
\text { Ecuador }\end{array}$ & 0.35 & 1 \\
\hline $\begin{array}{c}\text { Porcentaje población indígena * Mecanismo Institucional } \\
\text { * Participación movimiento indígena }\end{array}$ & $\begin{array}{c}\text { Ecuador, } \\
\text { Colombia } \\
\text { Venezuela }\end{array}$ & 0.53 & 0.92 \\
\hline \multicolumn{2}{|c|}{ Solución Completa } & 0.93 \\
\hline
\end{tabular}

Fuente: Elaboración propia.

\section{Primera configuración causal para un alto reconocimiento de derechos indígenas en la constitución: el caso de Bolivia}

La selección del caso boliviano tiene varios puntos interesantes. Primero está el alto porcentaje de población indígena, que es característica fundamental de sus procesos políticos (Landivar, 2015). En segundo lugar, el proceso constituyente se asemeja a lo ocurrido en países como Venezuela (1999) y Ecuador (2008), principalmente por el rol del ejecutivo como canalizador de la crisis y proceso constituyente. Por último, la participación del mundo indígena no solo se detuvo en sus organizaciones formales-partidarias, sino que también las organizaciones indígenas activamente presionaron el reconocimiento de derechos.

Las fuerzas políticas constituyentes comenzaron a surgir bajo un contexto de amplias y violentas agitaciones sociales y políticas, producto de lo que había sido la Guerra del Gas del octubre negro. Por tanto, la idea constituyente generó un proceso de cohesión social que culminó con un proyecto político liderado por el MAS (Movimiento al Socialismo) que levantó el año 2005 a su principal figura Evo Morales. Sin embargo, la llegada de Morales a la presidencia no estuvo exenta de polémicas, es incluso gracias a su antesala política lo que generó que el origen del proceso constituyente fuese difícil y con grandes negociaciones en el parlamento. Si bien la campaña de Morales apuntaba directamente a una Asamblea Constituyente, es gracias al amplio apoyo y participación del mundo indígena con el que logra establecer las bases del poder constituyente necesario para llevar a cabo uno de los procesos más participativos de América Latina. 
A diferencia de lo que se señalará para el caso de Colombia (1991), en el caso de Bolivia el mecanismo para generar el cambio en la constitución sí estaba contemplado en la Constitución vigente como ejercicio del poder popular. Por lo que el proceso constituyente no está marcado por una negociación para decidir la realización de éste, sino que para decidir aspectos esenciales del funcionamiento de la asamblea constituyente Pisarello (2014).

Respecto de la participación del mundo indígena, el caso de Bolivia presenta una historia de larga data de cómo las organizaciones indígenas tomaron posición de la construcción constitucional como camino a muchos años de exclusión política (Landivar, 2015; Pisarello, 2014; Landaeta, 2016). Por un lado, está la activa participación del movimiento indígena junto a los sindicatos de trabajadores y gremios en las distintas etapas del proceso constituyente. A través de los Consejos Educativos de Pueblos Originarios (CEPOs), la Confederación de los Pueblos Indígenas de Bolivia (CIDOB) y la Confederación Sindical Única de Trabajadores Campesinos de Bolivia (CSUTCB), se luchó por el reconocimiento de derechos indígenas y su participación (Landaeta, 2016). Esta construcción social que se da producto de una historia previa al proceso constituyente, tal como señala Landaeta (2016) fue clave para activar redes democráticas y de retroalimentación que validaron ampliamente el proceso:

"El fortalecimiento de las bases organizativas indígenas bajo el modelo de los ayllus, entidades territoriales administrativas tanto del territorio como de la sociedad aymara y quechua, y de los resguardos de Tierras Bajas, que hizo posible mantener cierto nivel de autonomías frente al Estado y al empresariado neoliberal. Mediante esta estrategia de representatividad social, las comunidades indígenas y los pueblos originarios tuvieron presencia, a través de sus organizaciones y dirigentes, siendo capaces de informar a las bases y actuar como interlocutores con las autoridades en las diferentes discusiones que se desarrollaron durante la discusión de la Nueva Constitución del Estado Boliviano" (Landaeta, 2016, p.348).

La organización se dio en las distintas etapas del proceso, desde el momento de negociación para el funcionamiento, hasta el referéndum de aprobación de la constitución. Sumado a lo anterior, también se establecieron escaños reservados especialmente para los pueblos indígenas, se delimitaron zonas de trabajo o cabildos con la sociedad para levantar la información, yenelreferéndum final participaron activamente movilizando a las comunidades para determinar si los derechos y la forma de redacción de aquellos era el pertinente. Por lo tanto, la fuerza del movimiento indígena no solo impacta de forma exitosa en apoyarel proceso constituyente, sino que además en transformarlo en un proceso participativo, plural y donde existe una incidencia directa del mundo indígena en la redacción de la nueva constitución. 


\section{Segunda configuración causal para un alto reconocimiento de derechos indígenas en la constitución: el caso de Colombia}

En la segunda configuración se observa el caso de Colombia (ver Tabla 4), país interesante producto de su bajo porcentaje de población indígena que rompe con la literatura que señala que el clivaje étnico sería fundamental para mayores niveles de reconocimiento en materia de derechos indígenas, pero también variables como el origen del proceso y la acción ciudadana funcionan para comparar con Chile.

En particular el caso colombiano fue uno de los primeros en América Latina en comenzar con un proceso constituyente, producto de una fuerte crisis social e institucional. Los estudiantes y profesores jugaron un rol fundamental en el proceso constituyente, a través del movimiento "Todavía Podemos Salvar Colombia" se promovió la necesidad de consultar, en papeletas separadas, a la ciudadanía sobre reformar la Constitución. Si bien el proyecto no estaba directamente encaminado a la búsqueda de soluciones para los pueblos indígenas, el movimiento ciudadano presionó de forma transversal por los diversos temas de la sociedad en ese momento.

El mecanismo institucional por el cual se realiza el proceso de cambio constitucional, y que la ciudadanía demandaba era un proceso de Asamblea Constituyente, producto de que la institucionalidad colombiana no contemplaba este tipo de mecanismo el movimiento fue pionero en generar presión a las autoridades parlamentarias, pero sobre todo al ejecutivo para realizar las modificaciones necesarias. En este sentido, el presidente Barco junto al apoyo de la Corte Suprema por medio de un decreto es quien apoya la medida institucional para comenzar con el proceso. Esto es interesante de destacar puesto que la crisis no solo generó cohesión ciudadana, sino que presiones suficientes para que el ejecutivo tomara posición con la demanda, tal como se señala en la teoría (reactivamente). Por su parte Negretto (2013) señala que "una de las características claves del proceso de diseño constitucional en Colombia fue el relativamente alto nivel de acuerdo observado en la Asamblea Constituyente, a pesar de que el proceso fue altamente descentralizado y que no se formó una coalición mayoritaria" (p. 179).

En cuanto a la participación de movimientos indígenas. El caso colombiano destaca por su alta representatividad, ya que incluyó miembros de todos los sectores de la sociedad, e incluso representantes de pueblos indígenas que nunca habían tenido representación en elecciones previas (Negretto 2013, p. 177). La asamblea se conformó por 74 miembros y contó con dos escaños reservados para pueblos indígenas, electos mediante un padrón especial y una representación proporcional a su población (3\% del total). Este hecho empujó a Colombia a conversar sobre un tema que hasta entonces había sido supeditado, 
los aportes sustanciales de los constituyentes sobre el reconocimiento de derechos son inevitable y promovieron dentro de la arena política la construcción de diseños flexibles para con los indígenas en años posteriores ${ }^{11}$.

\section{Observaciones Finales: ¿Qué considerar para el proceso constitucional chileno?}

Chile ha atravesado por distintas etapas de consolidación y cambio constitucional. Una primera etapa de fundación institucional en el año 1870, por otro lado, reformas que derivaron en la Constitución de 1833, para finalizar con una etapa de consolidación institucional con la Constitución de 1980, sobre todo si pensamos en los cambios realizados en el año 2005. Ahora bien, y en términos de derechos indígenas la situación cambia radicalmente, las distintas constituciones que han marcado los procesos políticos y sociales de Chile han olvidado completamente a los pueblos originarios, esto ha marcado según distintos autores (véase, Bengoa, 1999, 2007; Pairicán, 2013; Pinto ,2016; Figueroa, 2016, entre otros) una historia de relaciones conflictivas entre el Estado y las comunidades indígenas.

Destaca en el año 1989 en esta materia el llamado "Acuerdo de Nuevo Imperial" donde Patricio Aylwin junto a líderes de distintos pueblos indígenas firman un acuerdo en materia de derechos y reconocimientos. El entonces futuro presidente de la transición democrática señalaba en su programa de gobierno el compromiso de adoptar medidas destinadas al reconocimiento indígena y sus derechos en la constitución ${ }^{12}$.

A la fecha, dicho reconocimiento y compromiso ha estado ausente de las discusiones políticas. Lo que se ha reforzado como política de Estado ha sido un escenario de asimilación cultural en términos de políticas públicas, con leyes de rango no constitucional como por ejemplo la Ley Indígena 19.253. Los cambios realizados en la constitución del año 2005 en el gobierno de Ricardo Lagos no fueron la excepción y obviaron completamente los sucesos de conflicto entre Estado e indígenas que ocurrían en distintos puntos del país. La elocuencia con la que se ha desarrollado la política constitucional en materia indígena ha dejado a Chile en una de las peores posiciones a niveles comparados.

El actual proceso constituyente por el que está atravesando Chile está en un momento clave para subsanar una deuda histórica con los pueblos indígenas, sobre todo en materia de reconocimiento de derecho ${ }^{13}$. Frente a este escenario, y en base a los resultados del estudio se señalan 5 elementos fundamentales que el proceso constituyente chileno debe considerar en base a las experiencias de sus países vecinos.

11 Para ver más detalles sobre los efectos en diseño electoral ver: https://www.banrepcultural.org/biblioteca-virtual/credencial-historia/numero-146/la-constitucion-de-1991-y-los-indigenas

12 Para mas detalles: http://fundacionaylwin.cl/wp-content/uploads/2018/06/2.-Acuerdo-Nuevalmperial_ 1dic 1989.pdf

13 Previo a enviar la última versión de este artículo, el trámite de los escaños reservados para pueblos indígenas y afrodescendientes estaba en discusión en su segundo trámite en el Senado.https://www.biobiochile.cl/noticias/ nacional/chile/2020/1 1/23/al-limite-de-tiempo-senado-espera-resolver-esta-semana-escanos-reservados-para-pueblos-originarios.shtml 
En primer lugar, mirar las experiencias de reconocimiento de derechos indígenas en países donde el porcentaje de población indígena se asemeja al de Chile permitiría borrar del imaginario colectivo que esto se trata de un tema cuantitativo de cuántos son, por tanto, cuántos derechos se deben tener. El caso de Colombia en tal sentido es muy oportuno, pero también está lo ocurrido con Ecuador, Nicaragua, Venezuela y Paraguay por solo nombrar otros procesos constituyentes.

En segundo lugar, tampoco es condición necesaria para un alto reconocimiento de derechos indígenas que la crisis que apoya o que antecede al cambio de la constitución sea eminentemente cultural. Lo ocurrido en Colombia (1991) o Venezuela (1999) con amplios derechos indígenas reconocidos en sus constituciones, muestran que el cambio constitucional puede nacer como una crítica al modelo político, a la corrupción, a la desigualdad económica y de oportunidades, entre otros, y decantar en un apoyo irrestricto a los grupos minoritarios excluidos.

En tercer lugar, y lo que sin duda no puede estar fuera del aprendizaje del proceso constituyente Latinoamericano es la importancia del mecanismo institucional con el cual se debe desarrollar el cambio de la constitución. La asamblea/convención constituyente, muy bien explicado por Negretto (2018) es el camino que invita a la participación, democracia y a la representación plural de la sociedad, aspectos claves dentro del tema de los derechos indígenas. Las fuerzas que se deben convocar en una asamblea/convención constituyente permiten unificar a distintos sectores de la sociedad, tal como se ha señalado para los casos colombiano y boliviano. Para el caso chileno está recomendación ya es una realidad, sin embargo, hoy falta establecer mecanismos de participación ciudadana efectiva que sigan articulando las demandas sociales con los y las futuros/as constituyentes, de otra forma el proceso podría perder objetivos y su capacidad de legitimación se vería directamente afectada. En tal sentido, las experiencias de Bolivia, Ecuador y Colombia son aprendizajes vecinos que se deben abordar.

En cuarto lugar y en consideración con el segundo elemento clave, el rol del movimiento indígena no debe necesariamente tener una fuerza previa, sino que más bien, debe acaudalar fuerzas y cohesionarse políticamente en el momento que se activa el mecanismo de cambio constitucional. El ejemplo claro es lo que se señaló sobre Colombia (1991), la participación del mundo indígena desde los cimientos del proceso constituyente, las presiones para definir escaños reservados para pueblos indígenas y movilizar a las comunidades en procesos participativos generó un movimiento activo y robusto capaz de impactar en el reconocimiento indígena dentro de la constitución ${ }^{14}$.

En quinto y último lugar, el arquetipo que ofrece la experiencia comparada en América Latina - en especial los casos de Venezuela (1999), Ecuador (2008), Bolivia (2009) y Colom-

14 Para un análisis sobre cómo el movimiento social chileno ha tomado elementos indígenas vinculándolos a liberación y resistencia, leer el artículo de Pairicán: https://www.ciperchile.cl/2019/11/04/la-bandera-mapuche-y-la-batalla-por-los-simbolos/ 
bia (1991)- para el caso chileno, destaca el esfuerzo social por incluir la demanda indígena dentro del proceso de crisis social general, un tema ya abordado en el caso Chile. Luego que la capacidad del movimiento pueda absorber no sólo las demandas, sino también la fuerza del mundo indígena, aglutinando a los pueblos como miembros y participantes activos de la toma de decisión, esto puede verse ejemplificado, en acercar los procesos de cabildos ciudadanos a las comunidades principalmente rurales, así como entregar escaños reservados. Sobre esto último, se debe considerar que la aprobación de la nueva constitución con los derechos debe tener una importante repercusión deliberativa respecto de quienes vivirán directamente los efectos de estos nuevos derechos culturales.

\section{Bibliografía}

Aguilar, G., Lafosse, S., Rojas, H., y Steward, R. (2010). The Constitutional Recognition of Indigenous Peoples in Latin America. Pace International Law Review, 2, (2), 44-104.

Bird, K. (2014). Ethnic quotas and ethnic representation worldwide. International Political Science Review, 35(1), 12-26. https://doi.org/10.1177/0192512113507798

Cheibub, J. (2007). Presidentialism, Parliamentarism, and Democracy. Cambridge, USA: University Press.

Bengoa, J. (1999). Historia de un Conflicto. Los Mapuche y el Estado Nacional durante el siglo XX. Santiago, Chile: Planeta.

. (2003). Relaciones y arreglos políticos y jurídicos entre los estados y los pueblos indígenas en América Latina en la última década. Santiago, Chile: División de Desarrollo Social.

. (2007). La emergencia indígena en América Latina. Madrid, España: Fondo de cultura económica.

Epstein, J., Duerr, D., Kenworthy, L.., y Ragin, Ch. (2008). Comparative Employment Performance: A Fuzzy-Set Analysis. In Method \& Substance in Macro Comparative Analysis, pp. 63-90. New York, USA: Palgrave Macmillan.

Figueroa, Verónica. (2006). El multiculturalismo y los movimientos indígenas como marco de las políticas públicas y las estrategias de desarrollo. Documentos de Trabajo, (9), Santiago, Chile: Instituto de Asuntos Públicos de la Universidad de Chile.

. (2016). Pueblos indígenas y consolidación democrática: desafíos para un reconocimiento constitucional de sus derechos en Chile. Revista ANALES. $7(10)$

. (2019). Governance and indigenous peoples: new challenges to their conceptualization and implementation. En Farazmand, A. (ed.) Global Encyclopedia of Public Administration, Public Policy, and Governance. Florida, USA: Springer International Publishing AG.

Foweraker, J. (1995). Theorizing social movements. London, UK: Pluto Press.

Fuentes, C. y Fernández, J. (2020). The four worlds of recognition of indigenous rights. Journal of Ethnic and Migration Studies. doi: 10.1080/1369183X.2020.1797478

Ghai, Y. (2006). La Asamblea Constituyente en la elaboración de la Constitución Política. Estocolmo, Suecia: International IDEA.

Galligan, D. y Versteeg, M. (eds.). (2013). Social and Political Foundations of Constitutions. Cambridge, UK: Cambridge University Press.

Gargarella, R. (2013). Latin American Constitutionalism 1810-2010, the engine room of the Constitution. Oxford, UK: Oxford University.

Ginsburg, T., Melton, J., y Elkins, Z. (2010). The Endurance of National Constitutions. Cambridge, UK: Cambridge University Press.

Kelsen, H. 1945). General Theory of Law and State. Nueva York, USA: Russell and Russell.

Knight, J. (1992). Institutions and Social Conflict. Cambridge, UK: Cambridge Uni- 
versity Press

Kymlicka, W. (2009). Las Odiseas Multiculturales: Las Nuevas Políticas Internacionales de la Diversidad. Barcelona, España: Paidós.

Krehbiel, K. (1991). Information and Legislative Organization. Ann Arbor, USA: University of Michigan Press.

Landaeta, A. (2016). Las organizaciones y comunidades indígenas en la conformación de la base social para la asamblea constituyente en Bolivia.: la experiencia de los consejos educativos de pueblos originarios (cepos) bolivianos. In Quiero F. \& Gajardo J. (Eds.), Por una Asamblea Constituyente Constitución: Mecanismos, procesos y contenidos para una nueva (pp. 341-354). Buenos Aires, Argentina: CLACSO. doi: 10.2307/j.ctvtxw27s.17

Lijphart, A. (1993). Constitutional Choices for New Democracies. Journal of Democracy, 2 (1) 72-84.

Mansbridge, J. (2003). Rethinking Representation. The American Political Science Review, 97(4), 515-528.

Martí, S., y Villalba, S. (2012). ¿Pocos, pero guerreros? Multiculturalismo constitucional en cinco países con población indígena minoritaria. Revista Uruguaya de Ciencia Política 21: 1-17. Recuperado de http://www.scielo.edu.uy/scielo. php? script=sci_arttext\&pid=S1 688-499X2012000200004\&lng=es\&nrm=iso

McAdam, D. (1996). Conceptual Origins, Current Problems, Future Directions. En D. McAdam, J D. McCarthy y Mayer N. Zald (eds.), Comparative Perspectives on Social Movements (pp. 23-40). Cambridge, UK: Cambridge University Press

Mella, E. (2007). Los mapuche ante la justicia. Criminalización de la protesta indígena en Chile. Santiago, Chile: Ediciones LOM.

Millaleo, S. (2019). ¿̇Para qué sirve una Constitución?: reflexiones sobre la inclusión constitucional de los pueblos indígenas. Revista de Derecho, 32(1), 29-50. doi:10.4067/S0718-09502019000100029

Moncusí, A. (2004). Entrevista en Revista Teína, revista electrónica de cultura y sociedad, "Sociedades Multiculturales" (3). Recuperado de: www.revistateina. es

Negretto, G. (2008). Political Parties and Institutional Design: Explaining Constitutional Choice in Latin America. British Journal of Political Science, 39 (1), 117 - 139. doi: https://doi.org/10.1017/S0007123408000446

(2012). Replacing and Amending Constitutions: The Logic of Constitutional Change in Latin America. Law \& Society Review, 46(4), 749-779. (2013). Making Constitutions: Presidents, Parties, and Institutional Choice in Latin America. Nueva York, USA: Cambridge Universtiy Press.

- (2018). Democratic constitution-making bodies: The perils of a partisan convention. International Journal of Constitutional Law, 16 (1), 254-279.

Nolte, D., y Schilling-Vacaflor, A. (eds.). (2012). New Constitutionalism in Latin America: Promises and Practices. Burlington, USA: Ashgate.

North, D. y Weingast, B. (1989). Constitutions and Commitment: The Evolution of Institutional Governing Public Choice in Seventeenth Century England. The Journal of Economic History,..49(4), 803 - 832.

Pairicán, F. (2013). Rebelión en Wallmapu. Antecedentes de un levantamiento indígena en el Chile actual. Rebelión en Wallmapu. Resistencia del pueblo nación mapuche, (pp.10-15). Santiago, Chile: Editorial aun creemos en los sueños.

Pérez Liñán, A. (2010). Presidential Impeachment and the Political Instability in Latin America. Cambridge, UK: Cambridge University Press.

Pinto, J. (2016). Pueblo mapuche, 1810-2015. Dos siglos de lucha por justicia, reconocimiento y respeto. En: Namuncura, D., Pinto, J., Pairicán, F. y otros (eds). Nueva Constitución y Pueblos Indígenas, pp. 65-103, Santiago, Chile: Pehuén Editores.

Pisarello, G. (2014). Procesos Constituyentes. Caminos para la ruptura democrática. Madrid, España: Trotta.

Ragin, Ch. (2008). Redesigning social inquiry: Fuzzy sets and beyond. Chicago, USA: University of Chicago Press.

Salinas, S. (2005). Construcción identitaria en el conflicto Mapuche: ᄅreencuentro con el mito del Weichafe? En Aranda, G. y Salinas, S. Conflictos de identidades y política internacional (pp. 297- 302), Santiago, Chile: Editores RIL

Scheppele, K. (2013). The Rule of Law and the Frankenstate: Why Governance 
Checklists Do Not Work. Governance, 26 (4), 559-562.

Schneider, C. y Wagemann, C. (2012). Set-theoretic methods for the social sciences: A guide to qualitative comparative analysis. Cambridge, UK: Cambridge University Press.

Tarrow, S. (1998). Power in Movement. Social Movements and Contentious Politics. Cambridge, UK: Cambridge University Press

Uprimny, R. (2014). The recent transformation of constitutional law in Latin America: trends and challenges. Texas Law Review, 89, 1587- 1609

Van Cott, D. (2000). The Friendly Liquidation of the Past. The Politics of Diversity in Latin America. Pittsburgh, USA: University of Pittsburgh Press. . (2001). Explaining Ethnic Autonomy Movements in Latin America, Studies in Comparative International Development, 35 (4).

- (2002). Movimientos in dígenas y transformación constitucional en los andes. Venezuela en perspectiva comparativa. Rev. Venez. de Econ. y Ciencias Sociales, 8 (3) 41-60

. (2005). From Movements to Parties in Latin America. The Evolution of Ethnic Politics. Cambridge, UK: Cambridge University.

Weingast, B. (1997). The Political Foundations of Democracy and the Rule of Law. American Political Science Review 91: 245-63.

Weingast, B. (2005). Self-Enforcing Constitutions. California; USA: Stanford University.

Weyland, K. (2006). Bounded Rationality and Policy Diffusion: Social Sector Reform in Latin America. Princeton, USA: Princeton University Press. doi:10.2307/j.ctt7rsvd

Young, I M. (1990). Justice and the Politics of Difference. Princeton, USA: Princeton University Press

Yrigoyen, R. (2000). The constitutional recognition of indigenous Law in the Andean Countries. En: Assies, W., G. Van der Haar y Hoekema (eds.): The Challenge of Diversity, Indigenous Peoples and Reforms of the State in Latin America. Amsterdam, Países Bajos: Thela Thesis.

- (2011). Los derechos de los pueblos indígenas a los recursos naturales y al territorio. Conflictos y desafíos en América Latina. En El derecho a la libre determinación del desarrollo, participación, consulta y consentimiento (pp. 103 - 146). Santiago, Chile: Icaria Editorial. 